\title{
Differential expression of DHHC9 in microsatellite stable and instable human colorectal cancer subgroups
}

\author{
F Mansilla ${ }^{1,5}$, K Birkenkamp-Demtroder, ${ }^{*, 5}$, M Kruhøffer', FB Sørensen ${ }^{2}$, CL Andersen', P Laiho ${ }^{3}$, \\ LA Aaltonen ${ }^{3}$, HW Verspaget ${ }^{4}$ and TF Ørntoft'
}

'Molecular Diagnostic Laboratory, Department of Clinical Biochemistry, Aarhus University Hospital/Skejby, Brendstrupgaardsvej 100, DK-8200 Arhus N, Denmark; ${ }^{2}$ Department of Pathology, Aarhus University Hospital, Århus, Denmark; ${ }^{3}$ Department of Medical Genetics, University of Helsinki, Biomedicum Helsinki, Haartmaninkatu 8, Finland; ${ }^{4}$ Department of Gastroenterology and Hepatology, Leiden University Medical Center, Leiden, The Netherlands

Microarray analysis on pooled samples has previously identified ZDHHC9 (DHHC9) to be upregulated in colon adenocarcinoma compared to normal colon mucosa. Analyses of 168 samples from proximal and distal adenocarcinomas using UI33plus2.0 microarrays validated these findings, showing a significant two-fold $(\log 2)$ upregulation of DHHC9 transcript $\left(P<10^{-6}\right)$. The upregulation was more striking in microsatellite stable (MSS), than in microsatellite instable (MSI), tumours. Genes known to interact with DHHC9 as H-Ras or N-Ras did not show expression differences between MSS and MSI. Immunohistochemical analysis was performed on 60 colon adenocarcinomas, previously analysed on microarrays, as well as on tissue microarrays with 40 stage I-IV tumours and 46 tumours from different organ sites. DHHC9 protein was strongly expressed in MSS compared to MSI tumours, readily detectable in premalignant lesions, compared to the rare expression seen in normal mucosa. DHHC9 was specific for tumours of the gastrointestinal tract and localised to the Golgi apparatus, in vitro and in vivo. Overexpression of DHHC9 decreased the proliferation of SW480 and CaCo2 MSS cell lines significantly. In conclusion, DHHC9 is a gastrointestinal-related protein highly expressed in MSS colon tumours. The palmitoyl transferase activity, modifying N-Ras and H-Ras, suggests DHHC9 as a target for anticancer drug design.

British Journal of Cancer (2007) 96, 1896-1903. doi:10.1038/sj.bjc.66038I8 www.bjcancer.com

Published online 22 May 2007

(c) 2007 Cancer Research UK

Keywords: DHHC9; immunohistochemistry; microarray; microsatellite instability

Colon cancer is among the most frequent cancers in the Western World with an overall 5 years survival around $50 \%$ (Lv et al, 2006). Early diagnosis is a key for cancer survival, as early-stage tumours have a better prognosis. Therefore, it is relevant to identify new biomarkers for early lesions. We identified DHHC9 as an early colon cancer biomarker both at the transcript and protein levels.

Two major molecular subgroups of colon cancer exist, microsatellite instable (MSI) and microsatellite stable (MSS) (Fearon and Vogelstein, 1990; Lengauer et al, 1997), where MSI tumours represent approximately $15 \%$ of the total incidence (Clark et al, 2004). Microsatellite instable tumours show mutations or epigenetic alterations in the mismatch repair genes that lead to alterations in microsatellite DNA (short repeated sequences of DNA). Increasing evidence suggests that MSI tumours are associated with better prognosis (Clark et al, 2004) and that patients with MSI may not benefit from fluorouracil-based adjuvant chemotherapy (Ribic et al, 2003; Benatti et al, 2005).

A differential behaviour of single molecules in MSS and MSI tumours may help understanding the molecular basis of these two subgroups and their associated prognostic and therapeutic

*Correspondence: Dr K Birkenkamp-Demtroder; E-mail: kbdr@ki.au.dk

${ }^{5}$ These authors contributed equally to this work.

Received 3 November 2006; revised 30 April 2007; accepted 30 April

2007; published online 22 May 2007 implications. In the present investigation, we analysed these two subgroups with regard to DHHC9 expression.

In our previous study, microarray expression profiling identified a subset of several hundred expressed sequence tags (ESTs) differentially expressed in pools of distal sporadic colon cancer (Dukes' stages A, B, C and D) when compared to pools of normal mucosa (Birkenkamp-Demtroder et al, 2002). One of these was EST AA232508, identified as part of the ZDHHC9 gene and strongly upregulated in all cancer stages (Birkenkamp-Demtroder et al, 2002). Very recently, the protein encoded by the ZDHHC 9 gene, DHHC9, has been characterised. DHHC9 is a 364 amino-acid membrane-bound protein characterised by its Asp-His-His-Cys (DHHC) sequence, within a cysteine-rich domain (Swarthout et al, 2005). DHHC9 forms a complex with a Golgi-associated protein of $16 \mathrm{kDa}$, GCP16. This DHHC9-GCP16 complex has been associated with a protein fatty acyltransferase (PAT) activity, with in vitro specificity for H-Ras and N-Ras (Swarthout et al, 2005). Palmitoylation of Ras increases the affinity of farnesylated Ras for membranes (Dudler and Gelb, 1996), where Ras can be stably anchored (Berthiaume, 2002). Palmitoylation also facilitates Ras transport (Choy et al, 1999; Apolloni et al, 2000), which contributes to its biological function in signal-transduction pathways.

In this study, we analysed the expression of DHHC9 mRNA in 168 colorectal adenocarcinomas sampled in Denmark, The Netherlands and Finland, using microarrays. We also studied the protein 
expression pattern in more than a hundred samples including formalin-fixed paraffin-embedded (FFPE) sections and tissue microarrays (TMAs) using a monospecific polyclonal antibody raised against DHHC9. In vitro studies of COS7 cells overexpressing DHHC9 and colocalisation studies on FFPE specimens demonstrated a Golgi-restricted expression of DHHC9.

Overexpression of DHHC9 decreased the proliferation rate of SW480, CaCo2 and DLD-1 with enabled wnt signalling, significantly.

In conclusion, DHHC9 is strongly upregulated in adenocarcinomas of the gastrointestinal tract. The microsatellite status of colon adenocarcinomas distinguishes two highly different molecular subgroups of colon cancer regarding DHHC9 expression.

\section{MATERIALS AND METHODS}

\section{Clinical specimens}

We analysed 168 colorectal samples from patients in Denmark $(n=108)$, The Netherlands $(n=56)$, and Finland $(n=14)$, comprising 122 colon and 46 rectum cancers (Supplementary Table 1). Ten normal mucosa biopsies from the resection edge were collected from Danish patients. Informed consent was obtained from patients, and the local scientific ethical committees approved the study.

\section{Tissue handling, nucleic acid isolation and microsatellite analysis}

The specimens were obtained fresh from surgery and immediately snap-frozen in liquid nitrogen either in an SDS/guanidinium thiocyanate solution or Tissue-Tek OCT compound and subsequently frozen (Denmark), or dry-frozen (Finland and The Netherlands). Total RNA was isolated from the same piece of tissue using Trizol (Invitrogen, Carlsbad, CA, USA). RNA was checked on an Agilent Bioanalyzer and samples with a $28 \mathrm{~S}$ to $18 \mathrm{~S}$ ribosomal RNA ratio lower than 1.0 were excluded. The microsatellite status of the tumours was analysed as described previously (Suraweera et al, 2002; Kruhoffer et al, 2005). Tumours with lowfrequency MSI have similar clinical features as MSS tumours and were considered as such in this study.

\section{Gene expression analysis}

Labelling of RNA, hybridisation and scanning were performed as described (Dyrskjot et al, 2003). Biotin-labelled cRNA was prepared from $10 \mu \mathrm{g}$ of total RNA and hybridised to the Human Genome U133plus2.0 GeneChip (Affymetrix, Santa Clara, CA, USA) containing $>55000$ probe sets. The readings from the quantitative scanning were analysed by the Affymetrix Software MAS5.0. The resulting cell-files for all 178 samples were imported into ArrayAssist version 3.3 (Stratagene, La Jolla, CA, USA) and data were normalised using GC-RMA (Bolstad et al, 2003; Irizarry et al, 2003) as implemented in ArrayAssist.

\section{Statistical analysis}

Transcript values were expressed as median $\log 2 \pm$ s.d. A twotailed unequal variance $t$-test was used to determine the differences of DHHC9 expression level in normal colon mucosa vs MSS and MSI tumours, and between the MSS and MSI subgroups. Differences with a $P$-value $<0.05$ were considered as statistically significant.

\section{Real-time RT - PCR and normalisation}

Semiquantitative real-time RT - PCR was performed on 24 samples, four MSI and MSS samples and their corresponding matching normal mucosa as well as four additional MSI and MSS samples with non-matching mucosa. cDNA was synthesised as described (Andersen et al, 2004). RT-PCR analysis was performed in triplicates using TaqMan ${ }^{\circledR}$ probe assay ID Hs00211318_m1 (Applied Biosystems) as recommended by the manufacturers and run on a 7500 Fast Real-Time PCR system (Applied Biosystems, Foster City, CA, USA). Results were normalised against UBC, as described previously (Birkenkamp-Demtroder et al, 2002; Andersen et al, 2004).

\section{RACE and plasmid construction}

RACE was performed using a marathon ready colon adenoma carcinoma cDNA library (Clontech, Mountain View, CA, USA) with primers $5^{\prime}$-UTR-GGGGGACATGTACACTCTTCTTCGC and 3'-UTR-GCCACTGGAGGAAAGTGGAAGTCG.

Wild-type DHHC9 cDNA was PCR amplified from the cDNA library mentioned above using primers sense $5^{\prime}$-GGCAACA TGTCTGTGATGGTGGTGAG and antisense $3^{\prime}$-CTACTTCT CAGCTTCAGCTGCCTCC. DHHC9 cDNA was cloned into pcDNA 3.1 bidirectional (Invitrogen) and the DNA sequence was verified. Several normal and colon cancer patients' cDNAs were synthesised from total mRNA using Superscript ${ }^{\mathrm{TM}}$ cDNA synthesis kit (Invitrogen).

\section{Production of monospecific antibodies}

Polyclonal rabbit anti-DHHC9 antibodies \#EP384 were raised against the peptide CKGSWTGKNRVQNPYS (amino acids $261-$ 274), conjugated to KLH. Antisera were affinity purified against the peptide (Eurogentec, Seraing, Belgium).

\section{Immunofluorescence microscopy}

COS7 cells were cultured on RPMI 1640 medium supplemented with $10 \%$ FCS and $1 \%$ penicillin-streptomycin at $37^{\circ} \mathrm{C}$ and $5 \%$ $\mathrm{CO}_{2}$ and transfected using lipofectamine (Invitrogen), following the manufacturer's instructions. Transfected COS7 cells were fixed and permeabilised with cold methanol $\left(-20^{\circ} \mathrm{C}\right)$ at room temperature. Four micrometres FFPE tissue samples were deparaffinised and stained as described previously (Birkenkamp-Demtroder et al, 2005). Cells or FFPE specimens were stained with rabbit antiDHHC9 (1:100 cells, 1:250 FFPE) and mouse monoclonal anti58K ( $1: 50$ cells, 1:400 FFPE) (Abcam Ltd, Cambridge, UK). Secondary antibodies were goat anti-rabbit highly cross-adsorbed Alexa ${ }^{\mathrm{B}}$ Fluor 488 conjugated (Molecular probes; $1: 2000$ cells, FFPE) in combination with goat anti-mouse Alexa ${ }^{\mathbb{R}}$ Fluor 546 conjugated (Molecular probes, Eugene, OR, USA; 1:800 cells, 1:1600 FFPE). Cells were stained with DAPI for nuclear visualisation. Cells and FFPE tissues were mounted with Fluorescent Mounting Medium DakoCytomation, Glostrup, Denmark. Visualisation was performed using a Zeiss Axiovert fluorescence microscope with 1000 and $\times 400$ magnification or a Leica DMRS confocal microscope with a $64 \times$ HCX PlApo, 1.32 NA objective. Images were merged using Image $J$ and assembled with Adobe Photoshop 9.0.

\section{Cell proliferation assay}

Cell proliferation assays were performed on HCT15, LS174 TR4, HCT116, doxycycline-inducible $\left(1 \mu \mathrm{g} \mathrm{ml}^{-1}\right)$ DLD-1 (van de Wetering et al, 2002), $\mathrm{CaCo} 2$ and SW480 human colon cancer cells using CyQUANT $^{\circledR}$ NF (Invitrogen). Cell lines were grown as follows: HCT15 in RPMI 1640 medium, SW480 in D-MEM medium, HCT116 in McCoy medium, LS174 TR and DLD-1 in RPMI 1640 medium added HEPES. All cell lines were supplemented with $10 \%$ FCS and $1 \%$ penicillin/streptomycin. A total of 400-10000 cells per well were transfected as described above. Dye binding solution 
$(1 \times)$ was added after $48 \mathrm{~h}$ post-transfection and fluorescence intensities were measured using a Biotek FLEX800-TBIDE fluorescence microplate reader at excitation $485 / 20 \mathrm{~nm}$ and detection 528/20 nm. Assays were performed on six replicates per cell line and per plasmid construction.

\section{Immunohistochemistry}

Samples consisted of 60 FFPE biopsies from the superficial nonnecrotic part of adenocarcinomas and/or normal mucosa biopsies taken from the macroscopically normal resection margin previously analysed on microarrays (Birkenkamp-Demtroder et al, 2002; Kruhoffer et al, 2005). Biopsies from hyperplasic polyps, adenomas and normal mucosa from the gastrointestinal tract were taken through endoscopies.

The anti-DHHC9 antibody was applied to $4 \mu \mathrm{m}$ FFPE sections and stained, as described previously (Birkenkamp-Demtroder et al, 2005).

Tissue microarrays were obtained from BioCat GmbH (Heidelberg, Germany). A 'Colon Cancer Tissue Array COCA912-5-OL (UICC staging)' and a TMA with human tumours from multiple organ sites and normal specimens from the same sites (T82357135-BC) were stained for DHHC9 protein expression using a 1:250 dilution of the rabbit anti-DHHC9 antibody. Scoring of the TMAs was independently performed by two experienced investigators (FBS and KBD).

\section{Cell extraction, SDS gels and Western blots}

Transfected COS7 cells were harvested and lysed in lysis buffer (50 mu Tris- $\mathrm{HCl}$ pH 8.0, $150 \mathrm{~mm} \mathrm{NaCl}, 1 \mathrm{~mm}$ DTT, $1 \%$ Triton X-100 and protease inhibitor ROCHE complete, EDTA free). Total protein samples $(10-20 \mu \mathrm{g})$ were run on $12 \%$ SDS gels (Invitrogen) and transferred to nitrocellulose membranes. Membranes were blocked with $3 \% \mathrm{w} / \mathrm{v}$ non-fat powder milk PBS. The primary antibody was rabbit polyclonal anti-DHHC9 $(1: 100)$ and the secondary antibody goat anti-rabbit HRP conjugated (1:5000)
(DakoCytomation). The immunoreactive bands were visualised using ECL plus (Amersham biosciences, Piscataway, NJ, USA) and a UVP ChemiDoc-It, Imaging system (UVP Inc., Upland, CA, USA).

\section{RESULTS}

\section{Microarray and RT - PCR analysis}

Genome-wide expression profiling monitored the presence of several transcripts corresponding to DHHC9 and proteins described to interact with it, or sharing an evolutionary relationship (Fukata et al, 2004). We found the DHHC9 transcript to be overexpressed in colorectal cancer (CRC). We found a significant two-fold $\log 2$ increase $\left(P<10^{-6}\right)$ in transcript levels, when adenocarcinoma tissue samples were compared to normal mucosa samples (Figure 1A and Table 1). GCP16, GCP170 (Ohta et al, 2003), DHHC14 (Fukata et al, 2004), DHHC18 (Fukata et al, 2004) and N-Ras, genes known to be related to DHHC9, showed a moderate decrease or no change (Table 1 and Supplementary Figure 1). H-Ras transcription was upregulated in CRC (1.3-fold

Table I Summary of microarray transcript profiling

\begin{tabular}{|c|c|c|c|c|c|}
\hline & \multicolumn{2}{|c|}{ Median $\log 2$} & \multirow[b]{2}{*}{ Log 2 fold change } & \multirow[b]{2}{*}{$P$-value } & \multirow{2}{*}{$\begin{array}{c}\text { MSS/MSI } \\
P \text {-value }\end{array}$} \\
\hline & $\mathbf{N}$ & $\mathbf{T}$ & & & \\
\hline $\mathrm{DHHC9}$ & 6.3 & 8.3 & 2.0 & $<10^{-6}$ & $<10^{-13}$ \\
\hline GCPI6 & 8.8 & 8.7 & -0.1 & 0.06 & 0.07 \\
\hline GCPI70 & 8.6 & 8.2 & -0.4 & $<10^{-4}$ & 0.003 \\
\hline N-Ras & 6.7 & 6.7 & 0 & 0.964 & 0.61 \\
\hline H-Ras & 6.3 & 7.6 & 1.3 & $<10^{-4}$ & 0.008 \\
\hline $\mathrm{DHHCl} 4$ & 5.0 & 4.5 & -0.5 & 0.01 & 0.61 \\
\hline $\mathrm{DHHCl} 8$ & 5.2 & 5.3 & -0.1 & 0.18 & 0.002 \\
\hline
\end{tabular}

$\mathrm{MSI}=$ microsatellite instable; $\mathrm{MSS}=$ microsatellite stable. Data are given as median $\log 2$, fold change between normal $(N)$ and tumour $(T)$ and $P$-value.
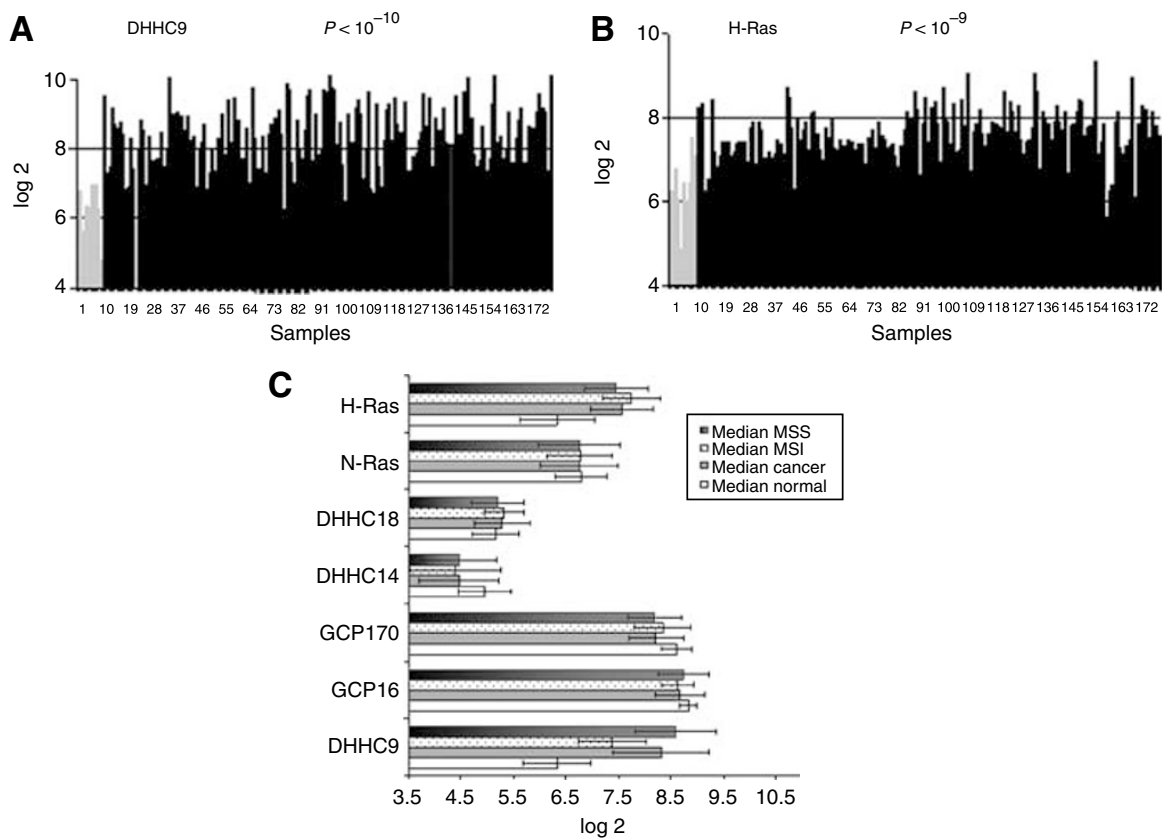

Figure I Microarray analysis of I 68 samples representing molecular subgroups of CRCs. Transcript expression level of $(\mathbf{A}) \mathrm{DHHC9}$ and $(\mathbf{B}) \mathrm{H}$-ras on the U 33 plus2.0 Gene Chip. The black bars correspond to CRC samples, the grey bars to normal mucosa. Expression values are given as log 2 values and all data are normalised. (C) MSS/MSI study. Median log 2 values and standard deviations of normal biopsies (median normal, $n=10$ ), CRC patients (median cancer, $n=168)$, MSI (CRC patients with MSI, $n=35)$, MSS (CRC patients with MSS, $n=1 \mid 8)$. 
change, $P<10^{-4}$ ) (Figure 1B and Table 1), confirming previously published data (Feng et al, 2001).

Out of 168 patient samples used in our microarray profiling study, 118 were identified as MSS, and 35 were MSI. The remaining 15 samples were of unknown status.

Data analysis pointed out that DHHC9 transcript overexpression differed significantly between MSS and MSI tumours $\left(P<10^{-13}\right)$, as depicted in Figure 1C. The MSS tumours (median $\log 2$, 8.6) showed a 2.3 -fold $\log 2$ increase $\left(P<10^{-7}\right)$ of DHHC9 transcript when compared to normal mucosa, while MSI tumours (median $\log 2,7.3)$ showed a 1.0 -fold $\log 2$ increase $\left(P<10^{-4}\right)$. In contrast to the DHHC9 transcript, the H-Ras transcript showed a 0.3 -fold $\log 2$ increase between MSI and MSS samples (Figure 1C).
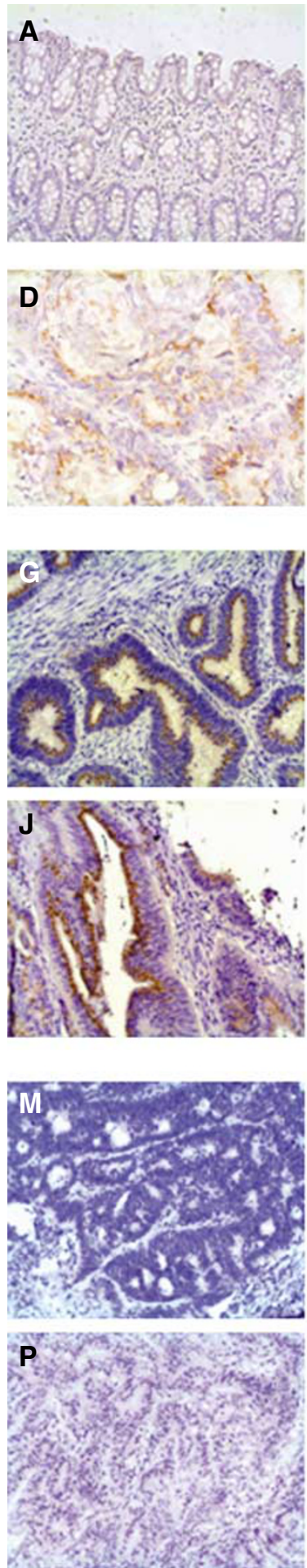
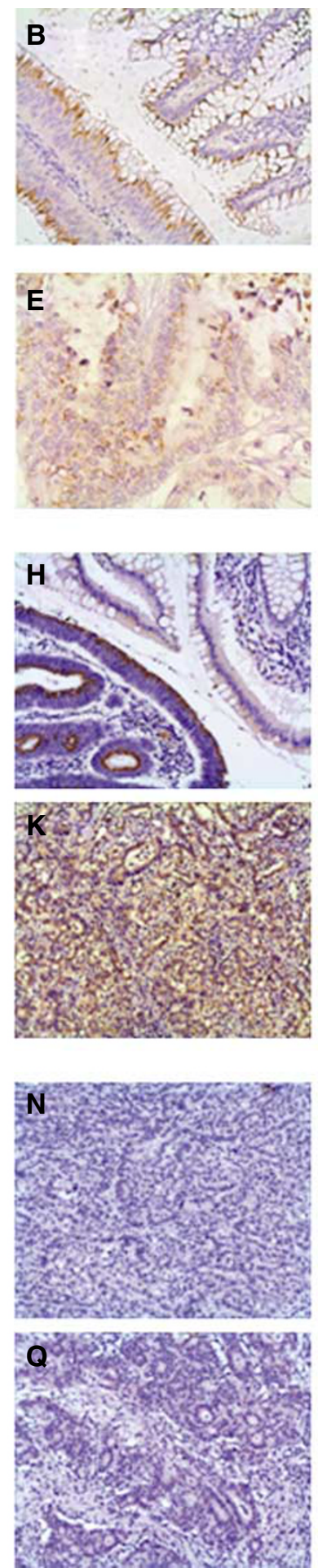
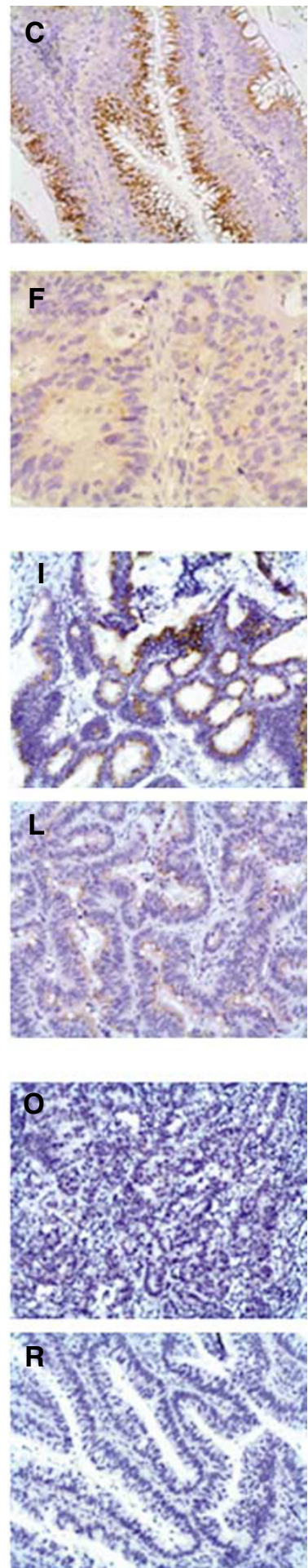

Figure 2 Immunohistochemical analysis of DHHC9 expression. (A) normal mucosa, (B) Dukes A adenocarcinoma adjacent to premalignant tissue, (C) Dukes A adenocarcinoma ( $\times 200)$, (D) Dukes D adenocarcinoma, $(\mathbf{E})$ lymph node metastasis and $(\mathbf{F})$ liver metastasis $(\times 400)$. DHHC9 protein expression in MSS vs MSI colon adenocarcinomas. Microsatellite stable tumours $(\mathbf{G}-\mathbf{L}), \mathrm{MSI}-\mathrm{H}$ tumours $(\mathbf{M}-\mathbf{R})(\times 200)$. 
Real-time RT - PCR was consistent with those data as ZDHHC9 transcript expression was only slightly increased in MSI tumours (median 1.1) but highly increased in MSS tumours (median 1.7) when compared to matching normal mucosa samples (median 0.4), as shown in Supplementary Figure 2.

\section{DHHC9 protein expression pattern in colon adenocarcinomas, distant metastases and TMAs}

Immunohistochemical analyses were performed on FFPE adenocarcinomas and their matching normal mucosa, all samples were previously expression profiled (Birkenkamp-Demtroder et al, 2005; Kruhoffer et al, 2005). Matching normal mucosa was either scored negative for DHHC9, or showed a very weak DHHC9 expression in the cytoplasm of the luminal part of the mucosa as shown in Figure $2 \mathrm{~A}$ and in more detail in Supplementary Figure 3.

DHHC9 protein was strongly upregulated in adenocarcinomas, thereby accumulating supranuclear, probably in the Golgi apparatus (Figure 2B and $\mathrm{C}$ )

To investigate whether DHHC9 was differentially expressed in MSS compared to MSI tumours, immunohistochemical analyses were performed on a subset of 34 colon adenocarcinomas with known MSS/MSI status, previously expression profiled on U133A2.0 arrays (Kruhoffer et al, 2005). The subset comprised 16 MSS and 18 MSI tumours and their matching normal mucosa. Eighty-one per cent of the MSS tumours were positive for DHHC9, showing a supranuclear accumulation of DHHC9 (Figure 2G-L), while $77 \%$ of the MSI tumours were negative for DHHC9 (Figure $2 \mathrm{M}-\mathrm{R})$. Supplementary Figure 3 shows the staining results of additional MSI (Supplementary Figure 3A-G) or MSS (Supplementary Figure $3 \mathrm{H}-\mathrm{M}$ ) tumours, and their matching normal mucosa. The scoring results are summarised in Table 2 , and in more detail in Supplementary Table 2.

Remarkably, upregulation of the DHHC9 protein was also detected in early lesions such as benign hyperplasic polyps and tubular and tubulo-villous adenomas (data not shown), as well as at premalignant sites in the colon mucosa (Figure $2 \mathrm{~B}$ and Supplementary Figure $3 \mathrm{~N}$ and $\mathrm{O}$ ) These sites were either located adjacent to, or in close proximity to, adenocarcinomas, for example, detected at the transformation site (Supplementary Figure $3 \mathrm{Q}$ ). Moreover, accumulation of DHHC9 protein was also identified in three lymph node metastases and 24 liver metastases analysed. An example showing an adenocarcinoma and two metastases from the same patient can be seen in Figure 2D-F.

To investigate whether differential expression of DHHC9 is stage dependent, we analysed a colon cancer TMA comprising adenocarcinomas of the UICC stages I-IV (Table 3). All 10 adenomas and $70-80 \%$ of the four UICC stages of adenocarcinomas and liver metastases stained positive for DHHC9. Eighty per cent of the normal mucosa samples were negative, only one was moderately expressing the DHHC9 molecule, as shown in Supplementary Figure 4.

In conclusion, DHHC9 is significantly upregulated in the majority of MSS tumours at both the transcript and protein level. Upregulation is detected at premalignant sites and in very early lesions and is independent of stage and grade.

\section{DHHC9 expression is confined to tumours of the gastrointestinal tract}

We analysed the DHHC9 protein expression pattern in 94 samples comprising 46 cancers from different organ sites and 46 normal tissues using a multiple cancer TMA. DHHC9 was either moderately or not expressed in normal tissues of the body, as shown in Supplementary Figure 5. Results are summarised in Supplementary Table 3. Most common cancers such as, bladder, prostate, breast, kidney, uterus, thyroid, lymph node, tongue or brain were completely negative for DHHC9 expression (Supplementary Figure 5). In contrast, all gastrointestinal adenocarcinomas from the colon rectum, small intestine and stomach showed a very strong positive Golgi-like staining for DHHC9 (Supplementary Figure 5B5, F7, F11 and G7). Moreover, analyses of additional 10 normal mucosa specimens derived from the gastrointestinal

Table 2 Immunohistochemical analysis of DHHC9 protein expression applying a 1:250 dilution of anti-DHCC9 antibody on 34 CRC tissue samples

\begin{tabular}{cccccccc}
\hline & Negative & Weak & Total & Moderate & Strong & Very strong & Total \\
\hline MSS & 3 & 0 & 3 & 3 & 5 & 5 & 13 \\
MSI & 8 & 6 & 14 & 1 & 1 & 4
\end{tabular}

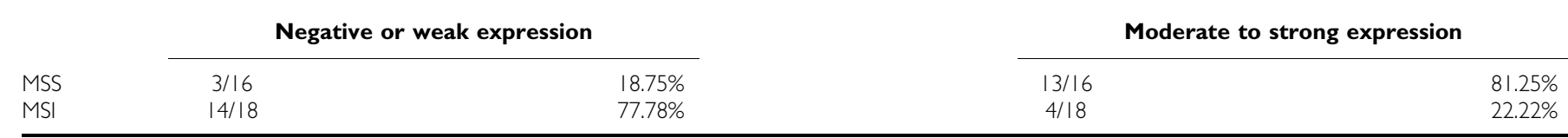

$\mathrm{CRC}=$ colorectal cancer; $\mathrm{MSI}=$ microsatellite instable; $\mathrm{MSS}=$ microsatellite stable. The intensity is classified as negative (comprising negative or weak) and positive (comprising moderate, strong or very strong).

Table 3 Immunohistochemical analysis of DHHC9 protein expression applying a 1:250 dilution of anti-DHCC9 antibody to a colon cancer TMA

\begin{tabular}{|c|c|c|c|c|c|c|c|}
\hline & Normal mucosa & Adenoma & ADC I & ADC II & ADC III & ADC IV & Liver metastases \\
\hline Average age (years) & 69 & 64 & 69 & 75 & 72 & 62 & 64 \\
\hline Female & 4 & 5 & 5 & 8 & 4 & 6 & 4 \\
\hline \multicolumn{8}{|l|}{ Staining } \\
\hline Strong & । & 3 & 5 & I & 3 & 2 & 4 \\
\hline Moderate & । & 7 & 3 & 7 & 5 & 5 & 4 \\
\hline
\end{tabular}

TMA = tissue microarray. The intensity of the positive cases is further classified as moderate or strong. 
tract of healthy people (oesophagus, stomach, small intestine, appendix, colon and rectum) were also negative for DHHC9 expression (data not shown).

\section{Subcellular localisation of DHHC9 in colon adenocarcinomas and transient overexpression of wild- type DHHC9}

Colocalisation studies using immunofluorescence microscopy were applied to FFPE tissue samples of adenocarcinomas with MSS status. DHHC9 colocalised with the Golgi marker 58K in the Golgi apparatus of the tumour cells, as shown in Figure $3 \mathrm{~F}-\mathrm{K}$.

The coding cDNA derived from Affymetrix probe set 222451_s_at (DHHC9) was amplified from a commercial colon adenocarcinoma cDNA library or normal and colon cancer total cDNAs. The sequences showed no mutations (data not shown). A $5^{\prime}$ - and $3^{\prime}$-UTR RACE amplified DHHC9 transcript variant 1 (NM_016032), proving the expression of a full-length transcript in CRC tissue.

Wild-type DHHC9 transiently overexpressed in COS7 cells colocalised with the Golgi marker $58 \mathrm{~K}$ to the Golgi apparatus (Figure $3 \mathrm{~A}$ and $\mathrm{D}$ ). This coincides with recent findings of Golgilocalised DHHC9 in the embryonic kidney cell line HEK293
(Swarthout et al, 2005) and the localisation in colon adenocarcinomas described above.

The specificity of the rabbit anti-DHHC9 antibody was confirmed by Western blot analysis, identifying a $36 \mathrm{kDa}$ band of recombinant wild-type DHHC9 (Figure 3E).

Interestingly, the apparent molecular weight was $5 \mathrm{kDa}$ lower than that of the calculated molecular weight of $40.9 \mathrm{kDa}$. This suggested that a cleavage of approximately $43-50$ amino acids may have taken place affecting the very basic $\mathrm{N}$-terminus of the protein, close to the predicted anchor signal (Lys35-Glu56). No bands were detected in the mock protein cell extract from COS7 cells transfected with an empty vector. There were no signs of cell morphological changes upon DHHC9 transfection.

\section{DHHC9 expression impacts proliferation of colon cancer cell lines}

Six colon cancer cell lines with MSS or MSI status were transiently transfected with DHHC9 or a mock empty vector. Proliferation was found to be decreased in SW480 and CaCo2 cell lines (MSS), while HCT15, LS174 TR4 and HCT116 (MSI) were not affected, as shown in Figure 4. All cell lines have an activated wnt-signalling pathway. To investigate the effect of DHHC9 on cell proliferation in a
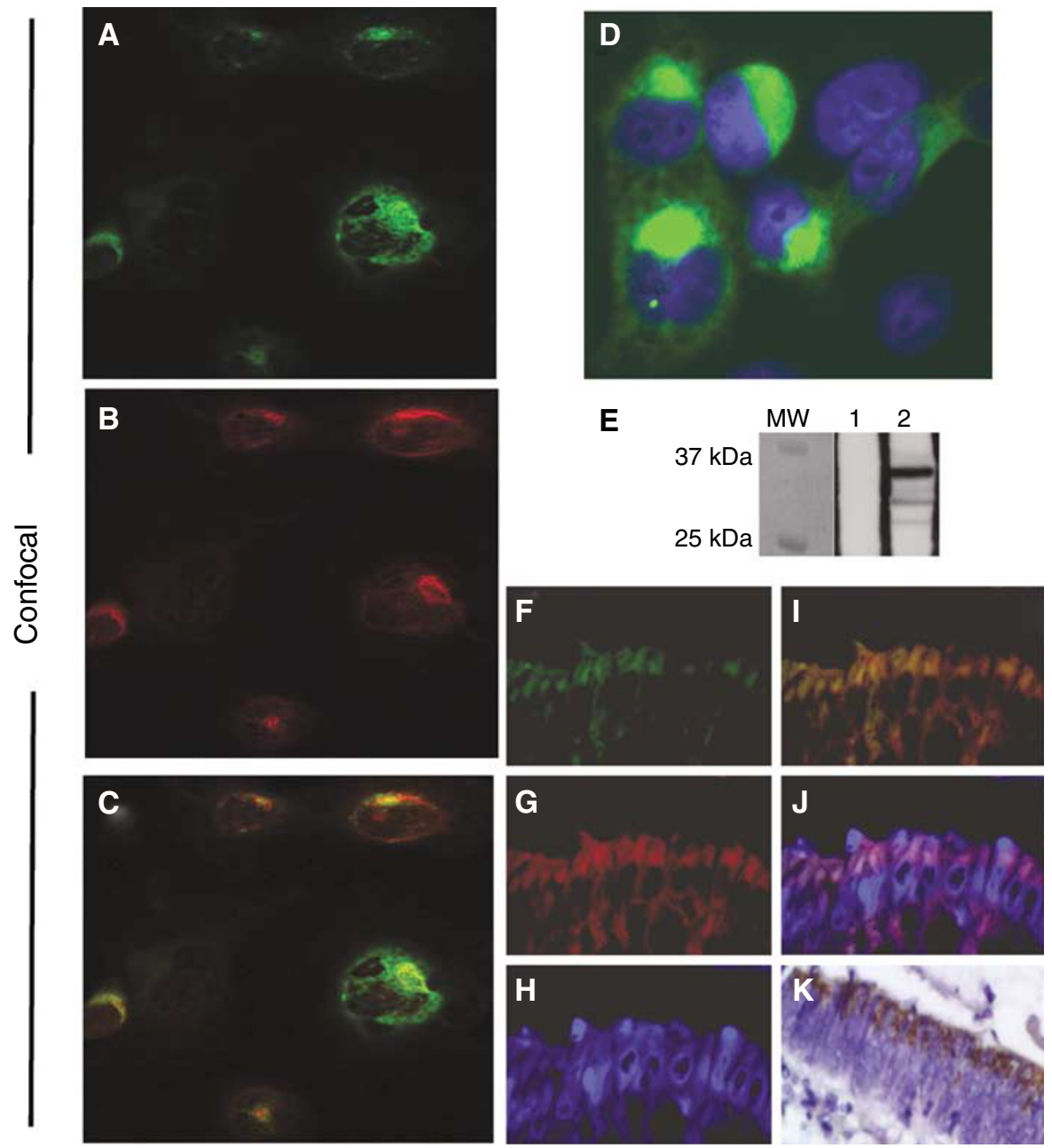

Figure 3 Immunostaining and Western blotting of transiently transfected COS7 cells and immunofluorescence applied to an MSS adenocarcinoma. (AC) Confocal microscope, (D) Zeiss Microscope: $\times 100$. (A) DHHC9, (B) endogenous 58K protein, (C) merged DHHC9 and 58K protein, (D) DHHC9 Golgi localisation detail. DAPI-stained nuclei are blue. (E) Western blotting of extracts from DHHC9-transfected COS7 cells (MW, molecular weight marker; I, cells transfected with an empty vector; 2, cells transfected with wild-type DHHC9). (F-K) IF, (F) DHHC9, (G) 58K protein, (H) DAPI, (I) merge $(\mathbf{F})$ and $(\mathbf{G})$; (J) merge $(\mathbf{F}),(\mathbf{G})$ and $(\mathbf{H}) ;(\mathbf{K})$ DAB staining of the same tumour. 


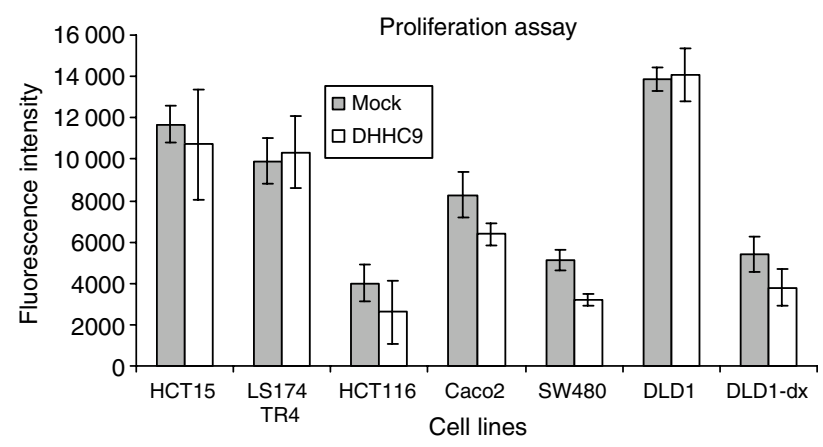

Figure 4 Proliferation assay. Several different cell lines with MSS (SW480, CaCo2) or MSI (HCTI5, HCTII6, LSI74 TR4, DLD-I) were transfected with $\mathrm{DHHC9}$ or a mock empty vector. Forty-eight hours posttransfection cells were added to I X dye binding solution, and fluorescence was measured. Only MSS cell lines SW480 and CaCo2 showed a significantly decreased proliferation rate. Interestingly, also DLD- I cells, with the wnt pathway switched off, showed a similar decreased proliferation rate.

cellular model, we used modified DLD-1 cells where the betacatenin/TCF4 activity can be disrupted by overexpression of dominant-negative TCF induced by doxycycline (van de Wetering et al, 2002). DHHC9 overexpression did not impact proliferation of DLD-1 cells with active wnt signalling. Remarkably, in addition to the previously described effects in SW480 and CaCo2, DHHC9 decreased the proliferation in cells with enabled wnt signalling compared to mock-treated cells.

\section{DISCUSSION}

Microarray analysis on 178 samples showed a significant upregulation of the DHHC9 transcript in individual colorectal tumour samples, when compared to normal mucosa. The upregulation of the DHHC9 transcript as well as the DHHC9 protein was mainly seen in MSS tumours.

The only published data on DHHC9 showed abundant transcript in the kidney, skeletal muscle, brain, lung and liver and moderate expression in the thymus, spleen and peripheral blood leucocytes based on a Northern blotting (Swarthout et al, 2005). In contrast, SAGE anatomic viewer at NCBI, based on small $10 \mathrm{bp}$ tags, showed a very low expression of the DHHC9 transcript in almost all the tissues tested (http://cgap.nci.nih.gov/SAGE). Our TMA-based data showed that, apparently, only tumours of the gastrointestinal tract were able to express the protein stably, and most of the other tissues seemed to be consistent with the transcriptional SAGE anatomic viewer profile.

Sporadic CRCs develop as a result of defects in pathways involving either chromosomal instability (Fearon and Vogelstein, 1990) or microsatellite instability (Lengauer et al, 1997).

Although the DHHC9 transcript was identified to be slightly upregulated in MSI tumours compared to normal mucosa, our results showed that DHHC9 transcript levels were significantly higher in MSS compared to MSI tumours.

DHHC9 has no repeats in the coding or promoter $5^{\prime}$-UTR regions. Sequencing of seven samples did not detect mutations in any of the amplified DHHC9 cDNAs from several patients (data not shown), leading to the conclusion that the coding sequence was not affected by mismatch repair failures.

It is well known that MSS tumours are genomic instable compared to MSS tumours.

Recently, genome-wide differences between MSS and MSI colorectal tumours have identified the loss of $\mathrm{Xq}$ in $31 \%$ of the MSS cases studied (Camps et al, 2006). We therefore investigated the ZDHHC9 Xq26.1 locus. Our own analyses of 15 laser microdissected colon adenocarinomas using Affymetrix Mapping $10 \mathrm{~K} \mathrm{SNP}$ arrays (Andersen et al, 2007) did not show loss or gain of chromosomal material at Xq26.1. There was no significant difference between male and female patients $(P=0.15)$. We conclude that the cause of DHHC9 upregulation and the difference in DHHC9 transcript level between MSS and MSI specimens is not directly related to a chromosomal imbalance at the Xq26.1 locus.

We have previously reported a differential gene expression in proximal compared to distal CRC tumours (Birkenkamp-Demtroder et al, 2005). In the present study, there was no significant expression difference between the proximal and distal colon with regard to the DHHC9 transcript. However, there was a tendency for MSI tumour samples to be originated in the right side, while MSS tumours are mostly left sided, as previously reported (Sugai et al, 2006).

DHHC9 transcripts were slightly upregulated in MSI tumours compared to normal mucosa. However, the protein itself was weakly expressed or absent in $77 \%$ of the MSI tumour samples. We may therefore consider a very rapid turnover of the protein, so it is not detectable, or there may be a tight regulation of the transcript, and consequently no translation will occur in MSI tumours.

Interestingly, proliferation of MSS cell lines SW480 and CaCo2, $48 \mathrm{~h}$ post-DHHC 9 transfection, showed a slight but significant decrease of the proliferation rate. In contrast, DHHC9 overexpression had no impact on the proliferation of MSI cell lines HCT116, HCT15, LS174 TR4 and DLD-1. All these cell lines have a constitutive active wnt-signalling pathway.

As expected, abrogation of wnt signalling significantly reduced proliferation in DLD-1 cells (van de Wetering et al, 2002). However, it cannot be excluded that part of the proliferation decreasing effect can be attributed to doxycycline treatment alone, as previously reported (Onoda et al, 2006). Surprisingly, the combination of wnt abrogation with a simultaneous DHHC9 overexpression yielded an even more dramatic reduction in proliferation than the wnt abrogation alone. These results may suggest a potential involvement for DHHC9 in the control of cellular proliferation. As a hypothesis, DHHC9 upregulation after a cellular damage may contribute to an arrest in the cell cycle. This arresting effect seemed to be associated to the MSS status, as only SW480 and $\mathrm{CaCo} 2$ transfected cells showed a decreased proliferation rate. Later on, other factors may probably counteract the antitumourigenic effect of DHHC9. Our data suggested that the potential effect of DHHC9 overexpression was independent of an active wnt-signalling pathway and had a synergistic effect upon abrogated wnt-signalling conditions. In addition, the highly tumourigenic, poorly differentiated SW480 cell line and the welldifferentiated $\mathrm{CaCo} 2$ showed a comparable decrease of the proliferation rate upon DHHC9 overexpression.

The function and location of the protein may suggest the implications of the DHHC9 expression in tumours. We localised the DHHC9 protein in the Golgi apparatus in both colon adenocarcinomas and transfected COS7 cells.

The DHHC9 protein has recently been described as a protein acyl transferase PAT specific for $\mathrm{N}$-Ras and $\mathrm{H}$-Ras (Swarthout et al, 2005). In vitro, DHHC9 and GCP16 are needed to carry out the palmitoyl transferase activity. Our transcript profiling identified DHHC9 transcript levels to be highly upregulated in MSS tumours compared to normal mucosa while GCP16 and N-Ras showed no differential expression in CRC. It is tempting to suggest that high DHHC9 levels of expression may have an impact on $\mathrm{N}$-Ras or H-Ras affecting its trafficking, modifying their acylation rate cycle regulation (Rocks et al, 2005) or affecting clusters of GTP bound, palmitoylated $\mathrm{H}-\mathrm{R}$ as and $\mathrm{N}-\mathrm{R}$ as, the so-called rasosomes (Rotblat et al, 2006).

In this context, proteins that are responsible for post-translational modifications are particularly suitable as potential anticancer drug targets (Leonard, 1997; Waddick and Uckun, 1998). Palmitoylation of N-Ras and $\mathrm{H}-\mathrm{Ras}$, a post-translational modification that takes place right after farnesylation, could become a new 
target. Unfortunately, although PAT activity is well known, the enzymes responsible for it are still elusive. However, the fact that DHHC9 has been postulated to be a PAT specific for both N-Ras and H-Ras, together with our results showing specific overexpression of the protein in gastrointestinal cancers already at the early stages of the disease, could be a first opening.

The role of DHHC proteins in cancer is just emerging. In this study, we bring forth a new perspective on ZDHHC9. We showed that $Z D H H C 9$ is significantly increased in CRC at the transcript and protein levels reflecting a significant difference between MSS and MSI tumours. In addition, the postulated specific PAT function of DHHC9, modifying N-Ras and H-Ras, suggests a very interesting potential as anticancer drug target.

\section{REFERENCES}

Andersen CL, Jensen JL, Orntoft TF (2004) Normalization of real-time quantitative reverse transcription-PCR data: a model-based variance estimation approach to identify genes suited for normalization, applied to bladder and colon cancer data sets. Cancer Res 64: 5245-5250

Andersen CL, Wiuf C, Kruhoffer M, Korsgaard M, Laurberg S, Orntoft TF (2007) Frequent occurrence of uniparental disomy in colorectal cancer. Carcinogenesis 28: $38-48$

Apolloni A, Prior IA, Lindsay M, Parton RG, Hancock JF (2000) H-ras but not K-ras traffics to the plasma membrane through the exocytic pathway. Mol Cell Biol 20: $2475-2487$

Benatti P, Gafa R, Barana D, Marino M, Scarselli A, Pedroni M, Maestri I, Guerzoni L, Roncucci L, Menigatti M, Roncari B, Maffei S, Rossi G, Ponti G, Santini A, Losi L, Di GC, Oliani C, Ponz de LM, Lanza G (2005) Microsatellite instability and colorectal cancer prognosis. Clin Cancer Res 11: $8332-8340$

Berthiaume LG (2002) Insider information: how palmitoylation of Ras makes it a signaling double agent. Sci STKE 2002: E41

Birkenkamp-Demtroder K, Christensen LL, Olesen SH, Frederiksen CM, Laiho P, Aaltonen LA, Laurberg S, Sorensen FB, Hagemann R, Orntoft TF (2002) Gene expression in colorectal cancer. Cancer Res 62: 4352-4363

Birkenkamp-Demtroder K, Olesen SH, Sorensen FB, Laurberg S, Laiho P, Aaltonen LA, Orntoft TF (2005) Differential gene expression in colon cancer of the caecum versus the sigmoid and rectosigmoid. Gut 54: 374-384

Bolstad BM, Irizarry RA, Astrand M, Speed TP (2003) A comparison of normalization methods for high density oligonucleotide array data based on variance and bias. Bioinformatics 19: 185-193

Camps J, Armengol G, del RJ, Lozano JJ, Vauhkonen H, Prat E, Egozcue J, Sumoy L, Knuutila S, Miro R (2006) Genome-wide differences between microsatellite stable and unstable colorectal tumors. Carcinogenesis 27: $419-428$

Choy E, Chiu VK, Silletti J, Feoktistov M, Morimoto T, Michaelson D, Ivanov IE, Philips MR (1999) Endomembrane trafficking of ras: the CAAX motif targets proteins to the ER and Golgi. Cell 98: 69-80

Clark AJ, Barnetson R, Farrington SM, Dunlop MG (2004) Prognosis in DNA mismatch repair deficient colorectal cancer: are all MSI tumours equivalent? Fam Cancer 3: 85-91

Dudler T, Gelb MH (1996) Palmitoylation of Ha-Ras facilitates membrane binding, activation of downstream effectors, and meiotic maturation in Xenopus oocytes. J Biol Chem 271: $11541-11547$

Dyrskjot L, Thykjaer T, Kruhoffer M, Jensen JL, Marcussen N, HamiltonDutoit S, Wolf H, Orntoft TF (2003) Identifying distinct classes of bladder carcinoma using microarrays. Nat Genet 33: $90-96$

Fearon ER, Vogelstein B (1990) A genetic model for colorectal tumorigenesis. Cell 61: $759-767$

Feng J, Hua F, Shuo R, Chongfeng G, Huimian X, Nakajima T, Subao W, Tsuchida N (2001) Upregulation of non-mutated H-ras and its upstream and downstream signaling proteins in colorectal cancer. Oncol Rep 8: $1409-1413$

Fukata M, Fukata Y, Adesnik H, Nicoll RA, Bredt DS (2004) Identification of PSD-95 palmitoylating enzymes. Neuron 44: 987-996

Irizarry RA, Bolstad BM, Collin F, Cope LM, Hobbs B, Speed TP (2003) Summaries of Affymetrix GeneChip probe level data. Nucleic Acids Res 31: e15

\section{ACKNOWLEDGEMENTS}

We thank Pamela Celis, Susanne Bruun and Jette Jensen for their excellent technical assistance as well as to Jeppe Praetorius, Institute of Anatomy, University of Aarhus, for confocal microscopy. The work was supported by grants from the John and Birthe Meyer Foundation, the Novo Nordisk foundation, the Danish Research Council, the University and County of Aarhus, the Nordic Cancer Union, and the Karen Elise Jensen foundation.

Supplementary Information accompanies the paper on British Journal of Cancer website (http://www.nature.com/bjc)

Kruhoffer M, Jensen JL, Laiho P, Dyrskjot L, Salovaara R, Arango D, Birkenkamp-Demtroder K, Sorensen FB, Christensen LL, Buhl L, Mecklin JP, Jarvinen H, Thykjaer T, Wikman FP, Bech-Knudsen F, Juhola M, Nupponen NN, Laurberg S, Andersen CL, Aaltonen LA, Orntoft TF (2005) Gene expression signatures for colorectal cancer microsatellite status and HNPCC. Br J Cancer 92: 2240-2248

Lengauer C, Kinzler KW, Vogelstein B (1997) Genetic instability in colorectal cancers. Nature 386: 623-627

Leonard DM (1997) Ras farnesyltransferase: a new therapeutic target. J Med Chem 40: $2971-2990$

Lv W, Zhang C, Hao J (2006) RNAi technology: a revolutionary tool for the colorectal cancer therapeutics. World J Gastroenterol 12: 4636-4639

Ohta E, Misumi Y, Sohda M, Fujiwara T, Yano A, Ikehara Y (2003) Identification and characterization of GCP16, a novel acylated Golgi protein that interacts with GCP170. J Biol Chem 278: $51957-51967$

Onoda T, Ono T, Dhar DK, Yamanoi A, Nagasue N (2006) Tetracycline analogues (doxycycline and COL-3) induce caspase-dependent and independent apoptosis in human colon cancer cells. Int J Cancer 118: $1309-1315$

Ribic CM, Sargent DJ, Moore MJ, Thibodeau SN, French AJ, Goldberg RM, Hamilton SR, Laurent-Puig P, Gryfe R, Shepherd LE, Tu D, Redston M, Gallinger S (2003) Tumor microsatellite-instability status as a predictor of benefit from fluorouracil-based adjuvant chemotherapy for colon cancer. $N$ Engl J Med 349: $247-257$

Rocks O, Peyker A, Kahms M, Verveer PJ, Koerner C, Lumbierres M, Kuhlmann J, Waldmann H, Wittinghofer A, Bastiaens PI (2005) An acylation cycle regulates localization and activity of palmitoylated Ras isoforms. Science 307: $1746-1752$

Rotblat B, Yizhar O, Haklai R, Ashery U, Kloog Y (2006) Ras and its signals diffuse through the cell on randomly moving nanoparticles. Cancer Res 66: $1974-1981$

Sugai T, Habano W, Jiao YF, Tsukahara M, Takeda Y, Otsuka K, Nakamura $S$ (2006) Analysis of molecular alterations in left- and right-sided colorectal carcinomas reveals distinct pathways of carcinogenesis: proposal for new molecular profile of colorectal carcinomas. J Mol Diagn 8: $193-201$

Suraweera N, Duval A, Reperant M, Vaury C, Furlan D, Leroy K, Seruca R, Iacopetta B, Hamelin R (2002) Evaluation of tumor microsatellite instability using five quasimonomorphic mononucleotide repeats and pentaplex PCR. Gastroenterology 123: 1804-1811

Swarthout JT, Lobo S, Farh L, Croke MR, Greentree WK, Deschenes RJ, Linder ME (2005) DHHC9 and GCP16 constitute a human protein fatty acyltransferase with specificity for $\mathrm{H}$ - and N-Ras. J Biol Chem 280: $31141-31148$

van de Wetering M, Sancho E, Verweij C, de Lau W, Oving I, Hurlstone A, van der Horn K, Batlle E, Coudrese D, Haramis AP, Tjon-Pon-Fong M, Moerer P, van den Born M, Soete G, Pals S, Eilers M, Medema R, Clevers $\mathrm{H}$ (2002) The beta-catenin/TCF-4 complex imposes a crypt progenitor phenotype on colorectal cancer cells. Cell 111: $241-250$

Waddick KG, Uckun FM (1998) Innovative treatment programs against cancer. I. Ras oncoprotein as a molecular target. Biochem Pharmacol 56: $1411-1426$ 